Article

\title{
'True' COVID-19 Vaccine Booster Hesitancy among Healthcare Workers: A Retrospective Observational Study in Singapore
}

\author{
Sky Wei Chee Koh ${ }^{1,2, *}$, Hwei Ming Tan ${ }^{1,2}$, Wayne Han Lee, ${ }^{1}$ Jancy Matthews ${ }^{1}$ and Doris Young ${ }^{2}$ \\ 1. National University Polyclinics, National University Health System, Singapore. \\ 2. Division of Family Medicine, Yong Loo Lin School of Medicine, National University of Singapore, Singa- \\ pore. \\ * Correspondence: sky_wc_koh@nuhs.edu.sg
}

Highlights

- $\quad 98.9 \%$ of HCW were fully vaccinated, $73.8 \%$ eligible had taken booster (December 2021)

- HCW less vaccine hesitant, median booster uptake 16 days compared to 1 st dose 39 days

- COVID-19 1st dose hesitant HCW were 3.6x more likely to be booster hesitant

- Medical, nursing $1.8 x$ more likely to receive booster earlier compared to admin staff

- No temporal relationship between booster uptake, legislation and infection numbers

\begin{abstract}
Background: COVID-19 booster uptake remained poor among healthcare workers (HCW) despite evidence of improved immunity against Delta and Omicron variants. While most studies used a questionnaire to assess hesitancy, this study aimed to identify factors affecting true booster hesitancy by examining actual vaccine uptake across time.

Method: COVID-19 vaccination database records among HCW working at 7 Singaporean public primary care clinics between January to December 2021 were extracted, with gender, profession, place of practice, vaccination type and dates. Time to booster was calculated from the date of vaccination minus date of eligibility. Chi-square test was used to compare relationship between 1st dose and booster hesitancy, Kaplan-Meier method and Log-rank test were adopted to evaluate differences in cumulative booster uptake. Multivariate cox regression was used to investigate predictors for timely booster vaccination. Vaccination rate was charted across time and corroborated with media releases pertaining to legislative changes.

Results: 877 of 891 (98.9\%) primary care HCW were fully vaccinated, 73.8\% of eligible HCW had taken the booster. HCW were less booster hesitant [median 16 (5-31.3) days] compared to the 1st dose [median 39 (13-119.3) days]. 1st dose hesitant HCW were more likely to be booster hesitant (OR=3.66, 95\%CI 2.61-5.14). Adjusting for sex, workplace and time to 1st dose, ancillary (HR=1.53, 95\%CI 1.03-2.28), medical ( $\mathrm{HR}=1.8,95 \% \mathrm{CI} 1.18-2.74)$ and nursing ( $\mathrm{HR}=1.8,95 \% \mathrm{CI} 1.18-2.37)$ received boosters earlier compared with administrative staff. No temporal relationship was observed between booster uptake, legislative changes and COVID-19 infection numbers.

Conclusion: Vaccine hesitancy among HCW had improved from booster to 1st dose, with timely booster vaccination among medical and nursing staff. Tailored education, risk messaging and strategic legislation might help to reduce delayed booster vaccination.

Trial Registration: This study was approved by the National Healthcare Group (NHG) Domain Specific Review Board (DSRB), Singapore on (Reg No. 2021/01120).
\end{abstract}

Keywords: COVID-19 vaccine; vaccine hesitancy; healthcare workers; primary care; general practice; Singapore 


\section{Introduction}

As of February 2022, more than 433 million individuals worldwide have been infected with Coronavirus Disease 2019 (COVID-19), with close to 6 million deaths since its emergence in late 2019. ${ }^{1}$ Vaccination is a key public health strategy because it has been shown to be effective in reducing risk of infection and severe disease. . $^{2,3}$ Vaccine hesitancy is defined by the World Health Organisation (WHO) as the delay in acceptance or refusal of vaccination despite availability. ${ }^{4}$ In the current COVID-19 pandemic, this has resulted in a significant global health threat with negative socio-economic and health effects to individuals and their communities. ${ }^{5}$

Healthcare workers (HCW) are at increased risk of exposure due to the nature of their work, thus achieving high vaccination rates with timely booster doses in this group is critical. As the first group to be vaccinated and imbued with adequate knowledge, HCW were often looked upon as highly trusted sources of guidance about COVID-19 vaccination. ${ }^{6} \mathrm{HCW}$ were therefore best positioned to share locally credible experiences to be role models for the rest of the community. However, levels of vaccine hesitancy among HCW were comparable to that of the general population across different countries. ${ }^{7}$ Hesitancy among HCW were lower among doctors, self-perceived high risk and involved with care for COVID-19 patients and improved confidence, with greater understanding of risks and side effects across time. .-11 $^{-1}$

An island city-state with close to 360,000 COVID-19 infections as of February 2022, Singapore managed to reduce community spread and kept its death rate low with a high vaccination rate of $91 \% .{ }^{12}$ This was achieved through legislation such as vaccine-differentiated safe management measures (VDS), evidence-based decision making, good communications and a strong primary care. Among HCW, vaccine hesitancy rates were low due to high self-perceived risk, similar to many studies performed abroad. ${ }^{13}$ Males, working in healthcare, ethnicity and age were associated with increased vaccination uptake. ${ }^{14}$ As new variants such as Delta and Omicron emerge, booster doses have been shown to confer greater protection by improving immunity for already fully vaccinated individuals. ${ }^{15,16}$ In Singapore, booster doses for mRNA COVID-19 vaccines were first introduced in September 2021. Those aged 12 and above are now eligible to receive their mRNA booster 5 months after completion of their primary vaccination series. However, only $58 \%$ of the total population have received their booster, compared to $67 \%$ among a public healthcare cluster. It would be interesting to observe response to booster vaccine uptake before it became a requirement to maintain fully vaccinated status for the purpose of VDS, which was announced in early January 2022. Given previous COVID-19 vaccination experience, we hypothesised lesser booster hesitancy among HCW with shorter lag time compared to previous vaccination, and uptake would be influenced by vaccinated related legislative changes. Hence, this study aims to examine true booster hesitancy and triangulate this with the timing of media announcements pertaining to legislative changes.

\section{Methods:}

The study was a retrospective cross-sectional study reviewing the prevalence, trend and factors affecting COVID-19 vaccine and booster uptake among employees in a healthcare cluster in Singapore.

\section{Study Population, Setting and Sampling}

COVID-19 vaccination and booster records of HCW working in a Singaporean public primary healthcare institution, comprising 896 staff from 7 primary care clinics and headquarters, were extracted from 1 January 2021 to 10 December 2021. This included physicians from Medical and Dental departments, nurses, allied health professionals and ancillary staff. Temporary staff, doctors doing clinical rotations and attachments were excluded in this study as it was not mandatory for their vaccination records to be updated in the database. Staff from pharmacy and diagnostics services within primary care clinics 
were not employed by the same public primary healthcare institution, and were hence excluded from this study.

There were 4 COVID-19 vaccine brands (Pfizer-BioNTech/Comirnaty, Moderna, CoronaVac, Sinopharm BIBP) approved for use in Singapore. The National Vaccination Programme recommended by the Expert Committee on COVID-19 Vaccination (EC-19V) recognised 2 mRNA (Pfizer-BioNTech/Comirnaty or Moderna) or 3 non-mRNA (CoronaVac, Sinopharm BIBP) vaccines as complete primary vaccination series.

HCW vaccination exercise within the institution started on 8 January 2021 with the Pfizer-BioNTech/Comirnaty vaccine, with adequate slots provided for HCW to undertake their vaccinations at their respective clinics across a 6-week duration. To ensure availability and accountability of vaccines, these slots were paired appointment dates for 2 consecutive COVID-19 vaccine doses 3-4 weeks apart (i.e., HCW who undertook COVID-19 vaccine dose 1 will have a guaranteed dose 2 appointment in 3-4 weeks).

Records of COVID-19 vaccination including brand and vaccination dates, sex, profession and place of practice were extracted anonymously from the Staff Surveillance System (S3), a one-stop database to track staff immunisations and surveillance used by public healthcare institutions in Singapore. It was an institution mandate to input and update vaccination records as this determined eligibility for work in high-risk areas. The cumulative vaccination rates were charted across time and corroborated with changes in legislative measures and media announcements.

For the purpose of this study, booster is defined as vaccination after completion of primary vaccination series. Based on EC-19V recommendations on 10 September 2021, booster should be received 6 months after mRNA primary vaccination series, or 3 months after 3rd dose for non-mRNA primary vaccination series. Invitations were sent to those all aged 60 and above or immunocompromised and booster vaccination commenced on 15 September 2021. This was extended to all aged 50 to 59 on 4 October 2021. Subsequently on 9 October 2021, booster vaccination was extended to all HCW in all clinics. Booster eligibility was brought forward to 5 months after 2nd COVID-19 vaccine dose (announced on 20 November 2021) due to evidence of waning antibodies. On 6 January 2022, it was announced that the booster dose would be required to maintain fully vaccinated status for the purpose of VDS for 270 days after the last dose of the primary vaccination series, from 14 February 2022 onwards, in Singapore.

To account for vaccine supply availability during initial vaccination service roll-out for the first dose of the COVID-19 vaccine, we defined COVID-19 1st dose vaccine hesitancy as failure of uptake within 6 weeks of vaccine introduction during the HCW vaccination exercise. This started on 8 January 2021. $\Delta 1$, defined by the time taken (in days) for 1st dose uptake from day of availability (8 January 2021), was calculated from HCW vaccination date. $\Delta 2$, time taken from 2 nd dose to 1st dose, was mostly fixed at 21-28 days as paired appointments were made to ensure vaccine availability. $\Delta 3$ was the number of days between COVID-19 dose 2 and booster vaccination. $\Delta 4$, defined as time taken (in days) for booster uptake from date of eligibility. This date of eligibility was defined as 6 months after 2nd COVID-19 dose (shortened to 5 months after 20 November 2021), or date of availability (15 September 2021 for HCW aged 60 and above, 4 October 2021 for HCW age 50-59 and 9 October 2021 for the rest of HCW), whichever was later. COVID-19 booster hesitancy was defined as failure to receive COVID-19 booster despite eligibility, as at 10 December 2021.

\section{Statistical Analysis}

Statistical analyses were performed with IBM ${ }^{\circledR}$ SPSS $®$ Statistics Version 28.0, R was used to calculate time between COVID-19 doses and date of eligibility for booster dose. $P$ value of $<0.05$ in two-sided test was considered as statistical significance. Descriptive statistics were performed and numerical variables were represented as mean $( \pm \mathrm{SD})$, median (IQR) or N(\%) for categorical variables. Chi square tests were used to evaluate COVID-19 booster hesitancy as of 10 December 2021 despite eligibility. The Kaplan-Meier method 
was used to estimate the cumulative coverage of COVID-19 vaccine and Log-rank tests were used to compare the difference across subgroups. Cox regression models were used to examine the predictors of delayed COVID-19 1st dose and booster vaccination. Finally, COVID-19 booster frequency was plotted against time and correlated with media announcements, vaccination changes and COVID-19 case numbers.

\section{Ethical considerations}

The study, analysis and publication of results was approved by the NHG Domain Specific Review Board (DSRB).

\section{Results}

98.5\% of all $891 \mathrm{HCW}$ had completed their COVID-19 primary vaccination series and were fully vaccinated (Figure 1). As of 10 December 2021, 73.8\% of eligible and fully vaccinated HCW had taken the booster while $26.2 \%$ were still hesitant, despite already being eligible for the booster vaccine. Demographics of HCW sampled and proportions in terms of sex, workplace and profession was depicted in Table 2.

Among all HCW who received their COVID-19 vaccination, $\Delta 1$ (in days) was $68.8 \pm$ $71.5,39(13,119.3)$ respectively. This was also calculated for $\Delta 2[24.2 \pm 11.8,21(21,24)], \Delta 3$ $[238.5 \pm 35.7,248(219,263)]$ and $\Delta 4[19.6 \pm 16.4,16(5,31.3)]$.

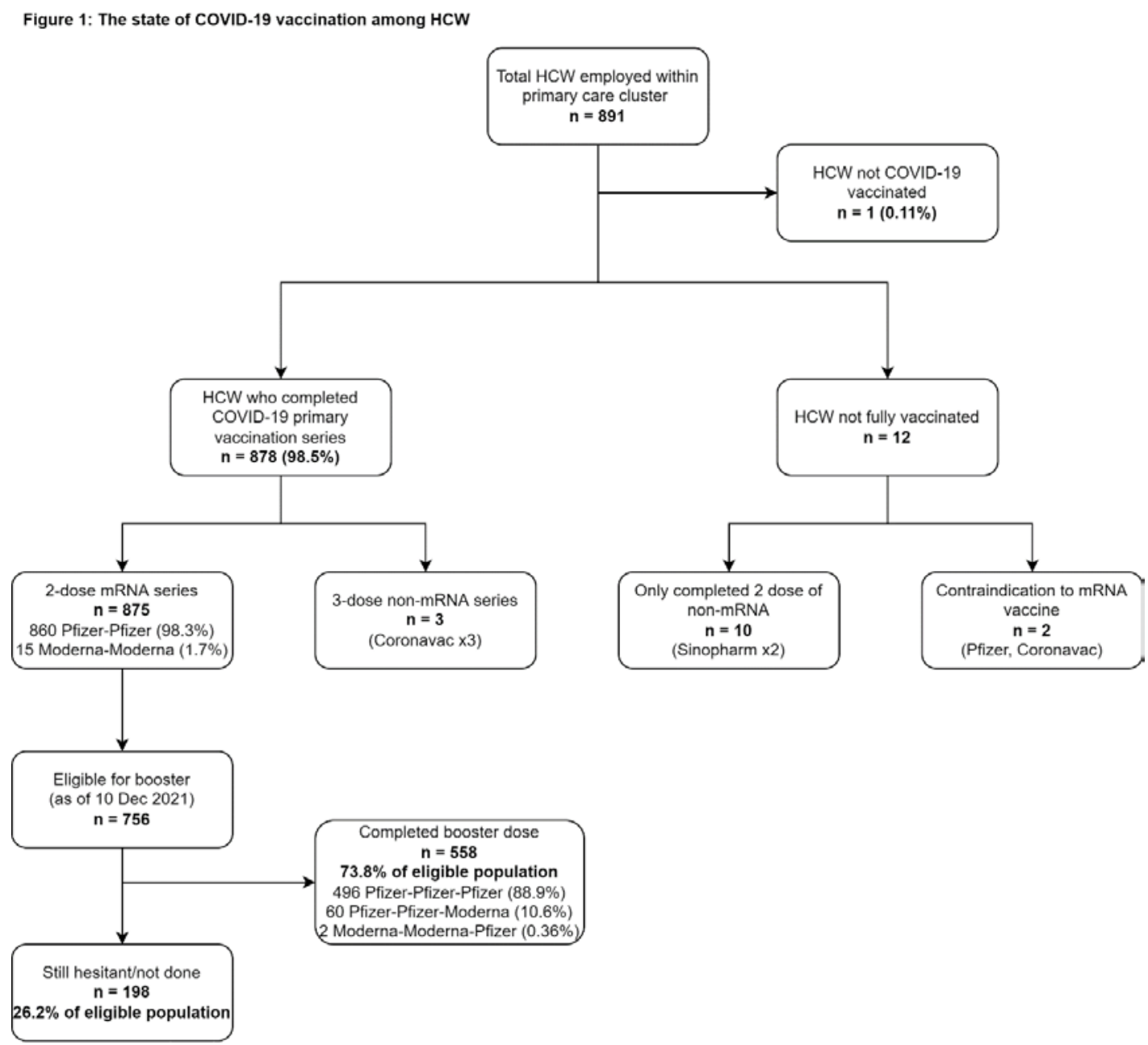

Figure 1. The state of COVID-19 vaccination among HCW.

HCW were split into 2 groups, hesitant (eligible but not boostered as of 10 December 2021) and not hesitant (vaccinated) towards the COVID-19 booster. There were no 
significant differences in booster hesitancy between sexes $(p=0.544)$, workplace $(p=0.134)$ or profession ( $p=0.299)$. Among $756 \mathrm{HCW}$, initial COVID-19 1st dose hesitancy was significantly associated with subsequent booster hesitancy $(\chi 2=59.9, \mathrm{P}<0.001)$. Compared to HCW who received COVID-19 1st dose during the HCW vaccination exercise, HCW who were 1st dose hesitant were 3.66 times more likely to be booster hesitant $(\mathrm{OR}=3.66,95 \%$ CI 2.61 - 5.14)

Table 2. Mean and median of delay in COVID-19 $1^{\text {st }}$ dose and booster uptake among HCW.

Table 2: Mean and median of delay in COVID-19 $1^{\text {st }}$ dose and booster uptake among HCW

\begin{tabular}{|c|c|c|c|c|c|c|c|c|}
\hline Study variables & \multicolumn{4}{|c|}{ COVID-19 vaccine $1^{\text {st }}$ dose delay (days) } & \multicolumn{4}{|c|}{ COVID-19 vaccine booster dose delay (days) } \\
\hline Sex & & & & $<0.001$ & & & & 0.782 \\
\hline Female & 757 (85.1\%) & $72.4(67.2,77.6)$ & $41(34.5,47.5)$ & & $466(83.5 \%)$ & $29(27.2,30.7)$ & $26(23.2,28.8)$ & \\
\hline Clinic & & & & 0.072 & & & & 0.044 \\
\hline Bukit Batok & $120(13.5 \%)$ & $59.6(46.2,73.1)$ & $20(10.7,29.3)$ & & $76(13.6 \%)$ & $29.3(24.9,33.7)$ & $24(12.3,35.7)$ & \\
\hline Headquarters & $138(15.5 \%)$ & $73.4(61.8,85.1)$ & $45(38.9,51.1)$ & & $87(15.6 \%)$ & $31.8(27.7,36.0)$ & $30(23.1,36.9)$ & \\
\hline Jurong & $148(16.6 \%)$ & $77.7(65.5,89.9)$ & $39(11,67)$ & & $94(16.8 \%)$ & $24.9(21.1,28.7)$ & $20(16.1,23.9)$ & \\
\hline Pioneer & $104(11.7 \%)$ & $55.3(43.6,66.9)$ & $31(13.5,48.5)$ & & $74(13.3 \%)$ & $25.9(21.3,30.5)$ & $18(10.3,25.7)$ & \\
\hline Queenstown & $73(8.2 \%)$ & $89.6(69.8,109)$ & $57(39.3,74.7)$ & & $32(5.73 \%)$ & $31.8(25.1,38.4)$ & $19(1.85,36.2)$ & \\
\hline Profession & & & & 0.019 & & & & 0.028 \\
\hline
\end{tabular}

+Category includes care coordinators, dieticians, medical social workers, physiotherapists, podiatrists, psychologists

${ }^{\wedge}$ Category includes call centre operators, financial counsellors, patient care and service associates

\#Category includes doctors and dentists

The Log rank test in Kaplan-Maier method (Table 2) showed that male sex and HCW profession were associated with shorter time to COVID-19 1st dose vaccination $(\mathrm{p}<0.05)$. Pairwise comparisons revealed that medical professionals have significantly shorter time to COVID-19 1st dose vaccination compared to their administrative $(p=0.007)$, ancillary $(\mathrm{p}<0.001)$ and nursing colleagues $(\mathrm{p}=0.021)$. This amounted to a mean of 10 , and a median of 18 days delay among administrative staff compared with medical HCW in receiving the COVID-19 booster.

Both HCW workplace and profession were significantly associated with differences in time to COVID-19 booster vaccination. Bukit Panjang and Jurong Polyclinic had significantly lower median time to booster compared to Choa Chu Kang ( $p=0.023,0.027)$, Headquarters $(p=0.027,0.028)$ and Queenstown $(p=0.039,0.019)$ Polyclinics. Shorter median time to COVID-19 booster were significantly noted among medical and nursing HCW, compared with their administrative $(\mathrm{p}=0.013,0.009)$ and allied health colleagues $(\mathrm{p}=$ $0.049,0.047)$. 
Table 3. Cox regression for time to COVID-19 booster among HCW.

Table 3: Cox regression for time to COVID-19 booster among HCW

\begin{tabular}{lccccc}
\hline Study variables & $\begin{array}{c}B \\
\text { coefficient }\end{array}$ & $p$ & HR & $\begin{array}{c}95 \% \text { Cl for HR } \\
\text { Lower }\end{array}$ & Upper \\
\hline Sex & & & & & \\
Female & -0.007 & 0.958 & 0.993 & 0.762 & 1.30 \\
Male & & & & & \\
& & & & & \\
Clinic & & & 1 & & \\
Bukit Batok & 0.278 & 0.108 & 1.32 & 0.941 & 1.85 \\
Bukit Panjang & -0.084 & 0.603 & 0.92 & 0.67 & 1.26 \\
Choa Chu Kang & -0.06 & 0.74 & 0.942 & 0.661 & 1.34 \\
Clementi & 0.367 & 0.08 & 1.44 & 0.957 & 2.18 \\
Headquarters & 0.258 & 0.098 & 1.29 & 0.954 & 1.76 \\
Jurong & 0.193 & 0.238 & 1.21 & 0.88 & 1.67 \\
Pioneer & -0.273 & 0.197 & 0.761 & 0.502 & 1.15 \\
Queenstown & & & & & \\
& & & 1 & & \\
Profession & & & 1 & & \\
Administration & 0.063 & 0.831 & 1.07 & 0.598 & 1.90 \\
Allied Health & 0.427 & 0.035 & 1.53 & 1.03 & 2.28 \\
Ancillary & 0.588 & 0.006 & 1.8 & 1.18 & 2.74 \\
Medical & 0.586 & 0.006 & 1.8 & 1.18 & 2.73 \\
Nursing & & & & & \\
Time to dose 1 $(\Delta 1)$ & -0.003 & 0.003 & 0.997 & 0.994 & 0.999 \\
& & & & & \\
\hline
\end{tabular}

$\mathrm{HR}=$ Hazard Ratio, $\mathrm{Cl}=$ Confidence Interval

Our Cox regression analysis yielded a significant $p$ value of 0.008 for omnibus tests of model coefficients at each step. After controlling for time to COVID-19 dose 1 vaccination, sex and workplace, profession was the key factor in affecting time to COVID-19 booster vaccination. Compared to administrative $\mathrm{HCW}$, ancillary $(\mathrm{HR}=1.53)$, medical $(\mathrm{HR}=1.8)$ and nursing $(\mathrm{HR}=1.8)$ staff were more likely to receive the COVID-19 booster earlier $(\mathrm{p}<0.05)$ (Table 3).

HCW COVID-19 booster vaccination rate was plotted against time; legislative changes and weekly COVID-19 infection numbers were highlighted as shown in Figure 4. Clinics were prompt in starting boosters for HCW, with increased uptake in the 4 weeks following 9 October announcement of HCW booster eligibility. Despite high weekly COVID-19 infection rates, booster uptake rate was low initially upon rollout in the first 4 weeks for HCW aged 60 and above, signifying no temporal relationship between the two. No significant increment of booster uptake was observed with the 20 November announcement of shortening of eligibility period to 5 months from 2nd dose. 


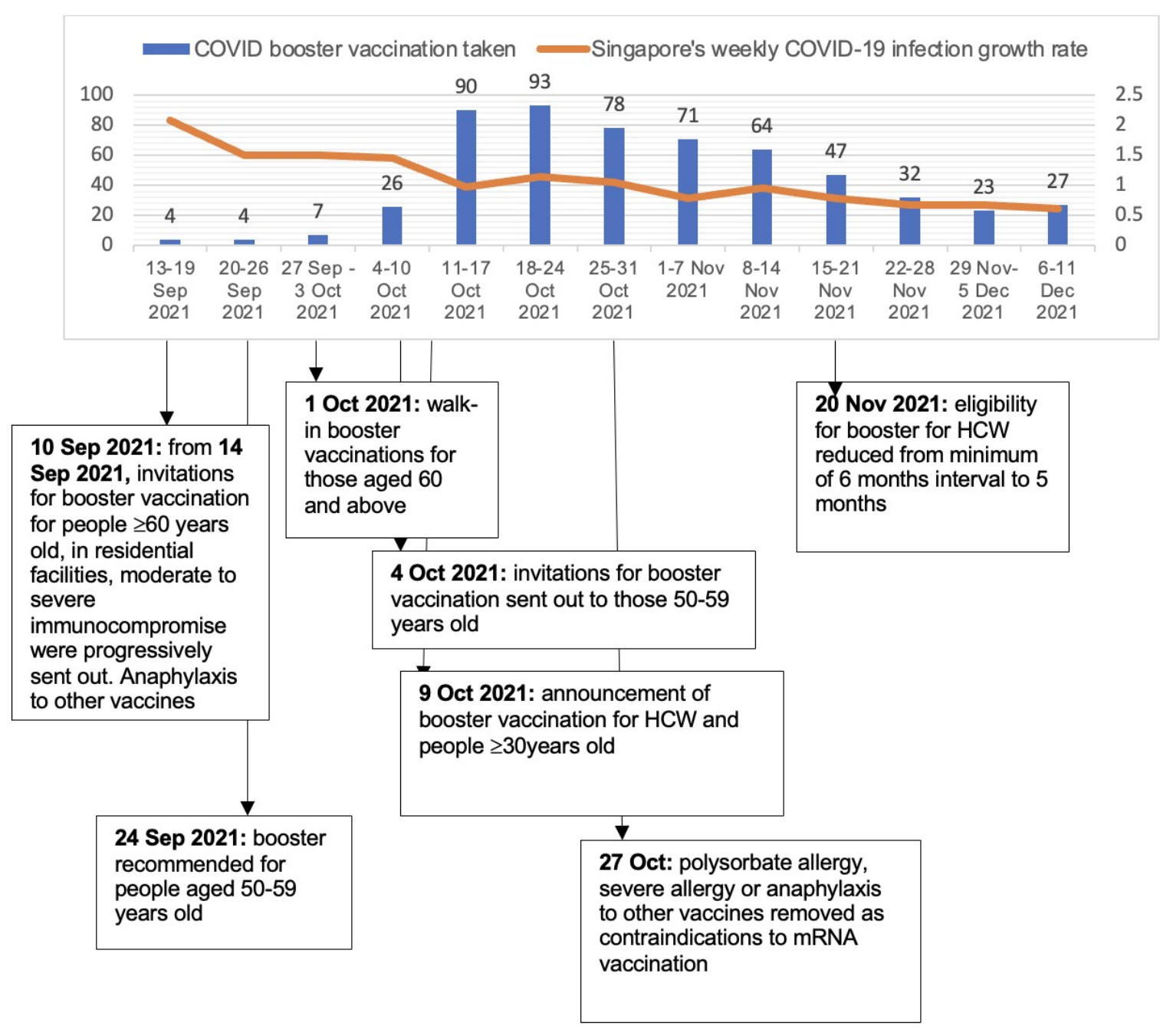

Figure 4. Timeline of COVID-19 booster uptake in relation to policy changes and local infection rates.

\section{Discussion}

This study was one of the first in measuring true vaccine hesitancy by examining absolute number and date of vaccine uptake from HCW vaccination database, instead of measuring hesitancy levels via questionnaires by research subjects conducted by numerous studies worldwide. This ensured the accuracy of COVID-19 dose 1, 2 and booster registration. The significant differences in timing of booster rollout for clinics might be explained by one clinic undergoing renovations during that time; on-site vaccination exercise was halted because of this.

Despite evidence and media releases on improved efficacy of Pfizer-Pfizer-Moderna over Pfizer-Pfizer-Pfizer COVID-19 booster combination, ${ }^{17}$ close to $90 \%$ of HCW opted for the Pfizer booster. We speculated that this was because of ease of accessibility to booster at all clinics (which only carried Pfizer), which corresponded to our previous study where we saw hesitant HCW ranking 'Constraints' as the lowest in the 5Cs hesitancy scale. ${ }^{13}$

Our study showed that COVID-19 booster delay was significantly shorter compared to initial 1st dose delay, possibly as confidence was boosted given numerous studies, accounts from family and experiences from their individual vaccinations. In particular, we found that the differences in sex for vaccine hesitancy had become insignificant for the booster dose; this was postulated to be due to developing evidence on safety of vaccines 
for pregnant and breastfeeding mothers, a concern also highlighted among female $\mathrm{HCW}^{18}$ and discovered in many hesitancy studies among HCW worldwide. ${ }^{7}$

The few papers published on booster hesitancy were in concordance with our study results. In a large UK study, initial 1st dose hesitancy increased the likelihood of booster hesitancy by 5 times..$^{19}$ This was noted among HCW within this study but to a lesser degree $(\mathrm{OR}=3.66)$, and the difference could be explained by the population group (HCW vs public) and setting (Singapore vs UK). It was also noted that booster hesitant individuals had low confidence or trust in authorities and vaccine messaging; ${ }^{20}$ this re-emphasized the need for targeted messaging to hesitant individuals to improve timely boosters to those who needed them the most.

Differences in vaccine hesitancy between HCW professions had been discovered previously in numerous studies, where medical and nursing professionals were found to be less hesitant compared to their ancillary and administrative colleagues. ${ }^{20,21}$ This paper was able to value-add by quantifying the differences in mean and median times to booster and showed significant differences after adjusting for confounders. This difference can likely be explained due to better vaccine knowledge, understanding of adverse effects and patient-facing roles adopted by doctors and nurses during the pandemic, a phenomenon also explored in previous studies.22,23

Limitations of this study included its selection bias of 1 primary care cluster in Singapore, and thus would not be representative of the whole primary care landscape. Compared to only $29.2 \%$ of the population having received the booster vaccination in midDecember 2021, achieving $62.6 \%$ booster vaccination among primary care HCW was definitely a commendable feat. Moreover, the rest of the healthcare cluster achieved this rate 1 month later in mid-January 2022, showing that primary care HCW might have been less hesitant. Cross-referencing with results from our previous study on the same cohort of $\mathrm{HCW}$, we speculated that this might be due to increased self-perceived risk to COVID-19, importance of protecting oneself and their family. 10,13

As COVID-19 vaccination and booster rollout was based on age cut-offs, it would be appropriate to analyse the impact of HCW age on vaccine hesitancy. Also, there were no guidelines for COVID-19 booster vaccination for recovered COVID-19 HCW who had completed their primary vaccination series. Knowing both HCW age and COVID-19 infection status would confer greater accuracy in determining eligibility date. However, both the extraction of age and previous COVID-19 status from the vaccination database did not receive ethics approval because this might make them easily identifiable and compromise on confidentiality. Because of this, we had to estimate the longest booster eligibility duration to be 61 days (between 10 October 2021 where booster was open to all HCW regardless of age and 10 December 2021 the end date of our study), where in actual fact this duration could have been longer due to earlier booster eligibility due to HCW age. Despite knowing that there were other factors not accounted for in our Cox regression, the strengths were that this study had zero dropout rate as all records were being extracted from the vaccination database. Despite a short follow-up time of 2 months, we were able to detect significant differences in time to booster between HCW groups. Future studies could focus on other factors (such as age and ethnicity, as discovered in a local study $)^{14}$ for a longer follow-up period to build a more robust Cox regression model to detect differences in other non-significant factors identified.

\section{Conclusion}

Findings suggested that COVID-19 booster vs initial 1st dose hesitancy among HCW had improved significantly, with close to $75 \%$ of HCW receiving booster in just 2 months upon its release. This would presumably be due to ease of accessibility, improved confidence with previous own experience of having received the vaccine and evidence and guidelines on safety profile. This study validated previous questionnaire studies on factors affecting vaccine hesitancy, showcasing significant delay in time to COVID-19 dose 1 among female HCW, and significantly shorter time to COVID-19 dose 1 and booster 
among medical and nursing HCW. Booster uptake was not temporally related to local COVID-19 infection rate. Future studies could examine age as a potential factor and further qualitative studies could help to explore the underlying factors correlated with vaccine hesitancy amongst HCW. Tailored education, improving awareness, risk messaging and strategic legislation might also help to reduce untimely booster vaccination.

Conflicts of Interests: The authors have no conflicts of interests to declare.

Declaration of funding support: This research did not receive any specific grant from funding agencies in the public, commercial, or not-for-profit sectors.

Trial Registration: This study was approved by the National Healthcare Group (NHG) Domain Specific Review Board (DSRB), Singapore on (Reg No. 2021/01120).

Disclosure of funding: No funding was provided

Competing interests: The authors declare that they have no known competing financial interests or personal relationships that could have appeared to influence the work reported in this paper.

Acknowledgements: Ban Hon Kim Kenneth (Assistant Professor, Department of Biochemistry, National University Singapore) for advice on statistical analysis, Lim Min Wei (Assistant Manager, Nursing Administration, National University Polyclinics) for data extraction.

Author's contributions: SWCK - Conceptualisation, Methodology, Investigation, Formal analysis, Writing (Original draft, Review \& Editing), Visualisation, Project Administration; HMT - Conceptualisation, Methodology, Formal analysis, Writing (Original draft, Review \& Editing); WHL Methodology, Investigation, Formal analysis, Data curation, Visualisation; JM - Conceptualisation, Methodology, Formal analysis; DY - Conceptualisation, Methodology, Supervision, Administration; All authors read and approved the final manuscript

\section{References}

1. COVID live - coronavirus statistics - worldometer [Internet]. [cited 2022 Feb 27]. Available from: https://www.worldometers.info/coronavirus/

2. Polack FP, Thomas SJ, Kitchin N, Absalon J, Gurtman A, Lockhart S, et al. Safety and efficacy of the bnt162b2 mrna covid-19 vaccine. New England Journal of Medicine. 2020 Dec 31;383(27):2603-15.

3. Moghadas SM, Vilches TN, Zhang K, Wells CR, Shoukat A, Singer BH, et al. The impact of vaccination on COVID-19 outbreaks in the United States. medRxiv. 2021 Jan 2;2020.11.27.20240051.

4. MacDonald NE, SAGE Working Group on Vaccine Hesitancy. Vaccine hesitancy: Definition, scope and determinants. Vaccine. 2015;33(34):4161-4164.

5. Harrison EA, Wu JW. Vaccine confidence in the time of COVID-19. Eur J Epidemiol. 2020 Apr;35(4):325-30.

6. Katzman JG, Katzman JW. Primary care clinicians as covid-19 vaccine ambassadors. J Prim Care Community Health. 2021 Dec;12:21501327211007024.

7. Sallam M. Covid-19 vaccine hesitancy worldwide: a concise systematic review of vaccine acceptance rates. Vaccines. 2021 Feb 16;9(2):160. 
8. Dror AA, Eisenbach N, Taiber S, Morozov NG, Mizrachi M, Zigron A, et al. Vaccine hesitancy: the next challenge in the fight against COVID-19. Eur J Epidemiol. 2020 Aug;35(8):775-9.

9. Gagneux-Brunon A, Detoc M, Bruel S, Tardy B, Rozaire O, Frappe P, et al. Intention to get vaccinations against COVID-19 in French healthcare workers during the first pandemic wave: a cross-sectional survey. Journal of Hospital Infection. 2021 Feb;108:168-73.

10. Kwok KO, Li K-K, Wei WI, Tang A, Wong SYS, Lee SS. Influenza vaccine uptake, COVID-19 vaccination intention and vaccine hesitancy among nurses: A survey. International Journal of Nursing Studies. 2021 Feb;114:103854.

11. Wang K, Wong ELY, Ho KF, Cheung AWL, Chan EYY, Yeoh EK, et al. Intention of nurses to accept coronavirus disease 2019 vaccination and change of intention to accept seasonal influenza vaccination during the coronavirus disease 2019 pandemic: A crosssectional survey. Vaccine. 2020 Oct;38(45):7049-56.

12. $\mathrm{MOH}$ - vaccination statistics [Internet]. [cited 2022 Feb 27]. Available from: https://www.moh.gov.sg/covid19/vaccination/statistics

13. Koh SWC, Liow Y, Loh VWK, Liew SJ, Chan Y-H, Young D. Covid-19 vaccine acceptance and hesitancy among singaporean primary healthcare workers [Internet]. In Review; 2021 Nov [cited 2022 Feb 20]. Available from: https://www.researchsquare.com/article/rs-983138/v1

14. Tan LF, Huak CY, Siow I, Tan AJ, Venugopalan PM, Premkumar A, et al. The road to achieving herd immunity: factors associated with Singapore residents' uptake and hesitancy of the COVID-19 vaccination. Expert Review of Vaccines. 2022 Jan 11;1-7.

15. Cameroni E, Bowen JE, Rosen LE, Saliba C, Zepeda SK, Culap K, et al. Broadly neutralizing antibodies overcome SARS-CoV-2 Omicron antigenic shift. Nature. 2021 Dec 23;1-9.

16. Lu L, Mok BW-Y, Chen L-L, Chan JM-C, Tsang OT-Y, Lam BH-S, et al. Neutralization of SARS-CoV-2 Omicron variant by sera from BNT162b2 or Coronavac vaccine recipients. Clinical Infectious Diseases. 2021 Dec 16;ciab1041.

17. Callaway E. Mix-and-match COVID vaccines trigger potent immune response. Nature. 2021 May 27;593(7860):491-491.

18. Berry SD, Johnson KS, Myles L, Herndon L, Montoya A, Fashaw S, et al. Lessons learned from frontline skilled nursing facility staff regarding COVID-19 vaccine hesitancy. Journal of the American Geriatrics Society. 2021;69(5):1140-6.

19. Paul E, Fancourt D. Predictors of uncertainty and unwillingness to receive the COVID-19 booster vaccine: An observational study of 22,139 fully vaccinated adults in the UK. The Lancet Regional Health - Europe. 2022 Mar;14:100317.

20. Paris C, Bénézit F, Geslin M, Polard E, Baldeyrou M, Turmel V, et al. COVID-19 vaccine hesitancy among healthcare workers. Infectious Diseases Now. 2021 Aug;51(5):484-7.

21. Klugar M, Riad A, Mohanan L, Pokorná A. Covid-19 vaccine booster hesitancy (Vbh) of healthcare workers in czechia: national cross-sectional study. Vaccines. 2021 Dec 6;9(12):1437.

22. Nguyen LH, Drew DA, Graham MS, Joshi AD, Guo C-G, Ma W, et al. Risk of COVID-19 among front-line health-care workers and the general community: a prospective cohort study. The Lancet Public Health. 2020 Sep;5(9):e475-83. 
23. Shaukat N, Ali DM, Razzak J. Physical and mental health impacts of COVID-19 on healthcare workers: a scoping review. Int J Emerg Med. 2020 Jul 20;13(1):40. 